\title{
Upregulation of miR-137 reverses sorafenib resistance and cancer-initiating cell phenotypes by degrading ANT2 in hepatocellular carcinoma
}

\author{
AI-QING LU ${ }^{1}$, BIN LV $^{1}$, FEI QIU ${ }^{2}$, XIAO-YUN WANG ${ }^{3}$ and XIAO-HUA CAO ${ }^{4}$ \\ Departments of ${ }^{1}$ Ultrasound and ${ }^{2}$ Gastrointestinal Surgery, ${ }^{3}$ Neonatal Intensive Care Unit and ${ }^{4}$ The First Intensive Care Unit, \\ The First People's Hospital of Jining, Jining, Shandong 272100, P.R. China
}

Received August 5, 2016; Accepted September 20, 2016

DOI: $10.3892 /$ or.2017.5498

\begin{abstract}
Hepatocellular carcinoma (HCC) is one of the most common malignancies worldwide. More than $80 \%$ of patients with HCC are not good candidates for curative surgical resection due to advanced liver cirrhosis caused by underlying chronic hepatitis virus (B or C) infection. Sorafenib, an oral multikinase inhibitor, is the only approved agent for the treatment of advanced HCC. Although, sorafenib currently sets the new standard for advanced HCC treatment, tumor response rates are usually quite low. An understanding of the underlying mechanisms for sorafenib resistance is critical. In the present study, we found that adenine nucleotide translocator 2 (ANT2) was upregulated in sorafenib-resistant HCC Huh7 cells (Huh7-R) and its overexpression promoted sorafenib resistance. ANT2 induced the formation of cancer-initiating cell (CIC) phenotypes and promoted metastasis-associated traits in the Huh7 cells. Silencing of miR-137 upregulated ANT2 protein expression in the Huh7 cells. miR-137 was downregulated in the Huh7-R cells, compared with that in the Huh7 cells and its restoration reversed sorafenib resistance in the Huh7-R cells. Restoration of miR-137 inhibited formation of CIC traits and attenuated the abilities of migration and invasion in the Huh7-R cells. Moreover, we demonstrated that high-intensity focused ultrasound (HIFU) in unresectable HCC upregulated serum miR-137. Combining HIFU and sorafenib may be a wise option for advanced and unresectable HCC.
\end{abstract}

\section{Introduction}

Hepatocellular carcinoma (HCC) is the most common liver cancer, accounting for $90 \%$ of primary liver cancers and is currently the third major cause of cancer-related deaths

Correspondence to: Dr Xiao-Hua Cao, The First Intensive Care Unit, The First People's Hospital of Jining, 6 Jiankang Road, Jining, Shandong, 272100, P.R. China

E-mail: caoxiaohuajn@163.com

Key words: hepatocellular carcinoma, ANT2, miR-137, HIFU worldwide (1). Although, recent progress in diagnostic and treatment technologies has improved survival, the long-term survival of HCC patients remains dismal. One of the reasons for the dismal prognosis is that current treatment cannot eliminate cancer-initiating cells (CICs) $(2,3)$. A comprehensive understanding of the molecular basis of CICs of HCC may contribute to the identification of novel therapeutic targets to improve patient outcome.

Sorafenib (Nexavar), a multiple kinase inhibitor, is the first and only drug that is clinically approved for patients with advanced HCC (4-8). The major target of sorafenib is serine threonine kinase Raf-1, which is involved in the Ras/Raf/MEK/mitogen-activated protein kinase signaling cascade $(9,10)$. In an in vitro kinase assay, sorafenib efficiently inhibited the activity of Raf-1 at a very low dose $\left(\mathrm{IC}_{50}\right.$ of $\left.6 \mathrm{nM}\right)(11,12)$. Other receptor tyrosine kinases are also suppressed by sorafenib, including vascular endothelial growth factor receptors 1,2 and 3, platelet-derived growth factor receptor and fibroblast growth factor receptor $(12,13)$. Although, sorafenib has exhibited survival benefits in large randomized phase III studies, the response rate to sorafenib is actually quite low $(2-3 \%)(4,14)$. In addition, therapeutic biomarkers that may predict the response to sorafenib are not currently available. Therefore, to improve the treatment response in HCC, it is important to identify the molecular mechanism of sorafenib resistance.

Adenine nucleotide translocator (ANT) which is abundant in the inner mitochondrial membrane, plays an important role in cellular energy metabolism by catalyzing the exchange of mitochondrial adenosine triphosphate for cytosolic adenosine diphosphate, thus, influencing mitochondrial bioenergetics (15). In addition, it is involved in the formation of the mitochondrial permeability transition pore complex that interacts with the $\mathrm{Bcl} 2$ family of proteins, contributing to mitochondrial-mediated apoptosis (16). Human ANT has 4 isoforms (ANT1, ANT2, ANT3 and ANT4), and the relative expression of these isoforms is dependent on the developmental stage, proliferation status and cell or tissue types. Among these isoforms, ANT2 is specifically expressed in proliferative and undifferentiated cells (16). It has been reported that ANT2 suppression by shRNA can exert anticancer effects in HCC through the regulation of different pathways (17-19). 
High-intensity focused ultrasound (HIFU) is based on the unique characteristic of ultrasound beams $(0.8-3.5 \mathrm{MHz})$, which can be focused at a distance from the radiating transducer (20). The accumulated energy at the focal region induces tissue necrosis of the targeted lesion without causing damage to the surrounding vital structures (20). The ability of inducing immediate cell death at a distance from the ultrasound source without the need for surgery or insertion of ablation instruments makes HIFU an attractive treatment option for HCC (20).

In the present study, we found that ANT2 was upregulated in sorafenib-resistant HCC Huh7 cells (Huh7-R) and its overexpression promoted sorafenib resistance. ANT2 induced the formation of CIC phenotypes and promoted metastasis-associated traits in the Huh7 cells. Silencing of miR-137 upregulated ANT2 protein expression in the Huh7 cells. miR-137 was downregulated in the Huh7-R cells, compared with the Huh7 cells and its restoration reversed sorafenib resistance in the Huh7-R cells. Restoration of miR-137 inhibited formation of CIC traits and attenuated the abilities of migration and invasion in the Huh7-R cells. Moreover, we demonstrated that HIFU in unresectable HCC upregulated serum miR-137. Combining HIFU and sorafenib may be a wise option for advanced and unresectable HCC.

\section{Materials and methods}

Patients. Between November 2012 and October 2014, 13 patients with HCC were enrolled in this clinical study. Four patients were not included in the present study since they either had $>4$ HCC foci $(n=2)$ or hepatic dysfunction (Child-Pugh class $\mathrm{C}, \mathrm{n}=2$ ). The present study was approved by the Ethics Committee of the First People's Hospital of Jining, and each patient signed an informed consent form at the time of enrollment.

Human HCC cell line. Huh7 cells were purchased from the Biochemistry and Cell Biology Institute of Shanghai, Chinese Academy of Sciences, within 3 months of the experiments. To obtain sorafenib-resistant Huh7 cells (Huh7-R cells), we treated Huh7 cells with increasing concentrations of sorafenib from $10^{7}$ to $10^{5} \mathrm{M}$. The established Huh7-R cells grew at a similar rate in the presence or absence of $10^{5} \mathrm{M}$ sorafenib for 3 days (data not shown). The $\mathrm{IC}_{50}$ (half maximal inhibitory concentration) of Huh7-R cells increased by 12-fold, compared with the Huh7 cells (data not shown). They were cultured in Dulbecco's modified Eagle's medium (DMEM) supplemented with $10 \%$ fetal bovine serum and antibiotics $(100 \mathrm{mg} / \mathrm{ml}$ penicillin, $100 \mathrm{U} / \mathrm{ml}$ streptomycin) in a $5 \% \mathrm{CO}_{2}$ incubator at $37^{\circ} \mathrm{C}$.

Western blotting. Whole cell lysates were subjected to SDS-polyacrylamide gel electrophoresis and transferred to polyvinylidene difluoride membranes (Bio-Rad, Berkeley, CA, USA). Then, proteins were probed with primary antibodies against human ANT2 (1:500; ab195630), RASSF1 (1:500; ab180801), SIRT3 (1:500; ab217319), NURR1 (1:500; ab60149), DLX2 (1:500; ab30339), ADRB2 (1:500; EP795Y), CD133 (1:500; ab19898), CD44 (1:500; ab157107), EpCAM (1:500; ab71916) and $\beta$-actin $(1: 500 ;$ ab8227; all from Abcam, Cambridge, MA, USA) overnight at $4^{\circ} \mathrm{C}$. Secondary antibodies (1:10,000; ab150077; Abcam) were used for $30 \mathrm{~min}$ at room temperature. The specific proteins were visualized by Odyssey ${ }^{\mathrm{TM}}$ Infrared Imaging System (Gene Company, Lincoln, NE, USA). $\beta$-actin expression was used as an internal control to show equal loading of the protein samples.

MTT assay. The proliferation of cells was assessed by the 3-(4,5-dimethylthiazol-2-yl)-2,5-diphenyltetrazolium (MTT) assay (Sigma-Aldrich, St Louis, MO, USA). The MTT analysis was performed as previously described (21-26). In brief, the cells were plated in 96-well plates in DMEM containing $10 \%$ fetal bovine serum at a density of $8 \times 10^{3}$ cells/well at $37^{\circ} \mathrm{C}$ in a $5 \% \mathrm{CO}_{2}$ incubator for $12 \mathrm{~h}$. Cells were transfected with ANT2-expressing plasmid or empty vectors and were then treated with sorafenib or dimethyl sulfoxide (DMSO) $\left(10^{-4}-10^{2}\right)$ for $24 \mathrm{~h}$. Alternatively, cells were transfected with pre-miR-137 or control miR (mock) for $24 \mathrm{~h}$. Then MTT solution $\left(5 \mathrm{mg} \cdot \mathrm{ml}^{-1}\right)$ was added to the wells $(20 \mu \mathrm{l} /$ well). Subsequently the plates were incubated in a cell incubator for $4 \mathrm{~h}$, then the supernatant was removed and $150 \mu \mathrm{l}$ of DMSO was added to each well. After incubation for $10 \mathrm{~min}$, the absorbance of each well was assessed using a Synergy ${ }^{\mathrm{TM}} 4$ Hybrid microplate reader (BioTek Instruments, Winooski, VT, USA) at a wavelength of $570 \mathrm{~nm}$, with the reference wavelength set at $630 \mathrm{~nm}$. The absorbance was directly proportional to the number of cells that survived.

Sphere formation assay. Cells $\left(10^{3} / \mathrm{ml}\right)$ in serum-free RPMI-1640/1 mM Na-pyruvate were seeded on $0.5 \%$ agar precoated 6-well plates. After 10 days, half the medium was replaced with fresh medium every third day. Single spheres were selected and counted.

Real-time PCR for microRNAs (miRs). Total RNA was extracted using the miRNeasy Mini kit and RNase-free DNase Set (Qiagen, Valencia, CA, USA) following the protocol provided by the manufacturer. The expression level of microRNA was analyzed using TaqMan MicroRNA Assay kit (Applied Biosystems) following the manufacturer's protocol.

Immunofluorescence staining. Immunofluorescence staining was performed as previously described (27). After transfection, the cells were fixed with $4 \%$ paraformaldehyde for $10 \mathrm{~min}$ and permeabilized with phosphate-buffered saline (PBS) containing $1 \%$ Triton $\mathrm{X}-100$ for $10 \mathrm{~min}$ at room temperature. Then, the coverslips were blocked with BSA and incubated with the primary antibodies against ANT2 (Abcam) overnight. The following day, the cells were incubated with the secondary antibodies (Abcam).

Bioinformatics method. The analysis of potential microRNA target sites was performed with the commonly used prediction algorithm, miRanda (http://www.microrna.org/).

Migration and invasion assays. Migration and invasion assays were performed as previously described (28).

Wound healing assay. Cells were seeded onto 6-well plates. Monolayers of cells were wounded with yellow pipette tips (volume range, $200 \mu \mathrm{l}$ ). After washing, the cells were 




Figure 1. Adenine nucleotide translocator (ANT2) is upregulated in sorafenibresistant Huh7 cells (Huh7-R cells) and its overexpression promotes sorafenib resistance in Huh7 cells. (A) Western blotting for ANT2 in Huh7 and Huh7-R cells. $\beta$-actin was used as a loading control. $n=3$. (B) Western blotting for ANT2 in Huh7 cells transfected with ANT2-expressing plasmids and empty vectors (mock). $\beta$-actin was used as a loading control. $n=3$. (C) An MTT assay was performed for cell viability in Huh7 cells. Huh7 cells transfected with ANT2-expressing plasmids and empty vectors (mock) were untreated or treated with sorafenib. (D) Western blotting for RASSF1, SIRT3, NURR1, DLX2 and ADRB2 in Huh7 cells transfected with ANT2-expressing plasmids and empty vectors (mock). $\beta$-actin was used as a loading control. $n=3$.

incubated in fresh culture medium. The wounded areas were photographed at 0 and $36 \mathrm{~h}$ using a Nikon inverted microscope.

Anoikis assays. Anoikis resistance was evaluated by seeding $7 \times 10^{4}$ cells in ultralow attachment plates (Corning Inc., Corning, NY, USA). After $24 \mathrm{~h}$ of anchorage-independent culture, cells were transfected as indicated and resuspended in $0.4 \%$ trypan blue (Sigma-ALdrich) and the viability of the cells was assessed using the CellTiter-Glo ${ }^{\circledR}$ Luminescent Cell Viability Assay (Promega, Madison, WI, USA). The cells were harvested with Triton X-100 lysis buffer at indicated times.

Statistical analysis. Data are presented as the mean \pm SEM. A Student's t-test (two-tailed) was used to compare differences between two groups $(\mathrm{P}<0.05$ was considered significant).
A

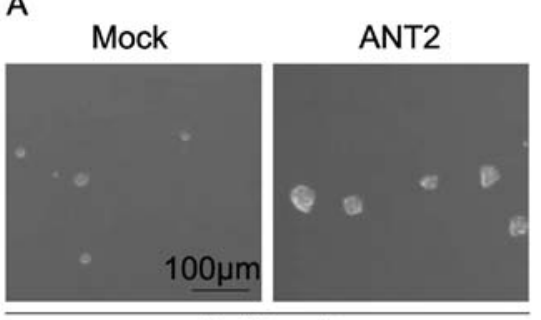

Huh7 cells

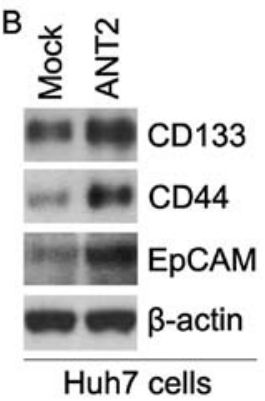

Huh7 cells

Figure 2. Adenine nucleotide translocator (ANT2) promotes the formation of cancer-initiating cell (CIC) phenotypes in Huh7 cells. (A) Sphere growth in Huh7 cells transfected with ANT2-expressing plasmids and empty vectors (mock). The data are reported as the number of spheres formed/1,000 seeded cells. $n=3$. (B) Western blotting for the protein expression of CD133, CD44 and EpCAM in Huh7 cells transfected with ANT2-expressing plasmids and empty vectors (mock). $\beta$-actin was used as a loading control. $n=3$.

\section{Results}

ANT2 is upregulated in sorafenib-resistant Huh 7 cells (Huh7-R cells) and its overexpression promotes sorafenib resistance in sorafenib-sensitive Huh7 cells. In order to detect whether sorafenib resistance is associated with ANT2 protein expression, we analyzed the protein expression of ANT2 in Huh7 and Huh7-R cells. The results revealed that the ANT2 protein was upregulated in the Huh7-R cells (Fig. 1A). To identify the role of ANT2, we ascertained whether ANT2-expressing plasmids could cause stable expression of the ANT2 protein in the Huh7 cells. The results showed that the ANT2 protein was significantly increased by ANT2-expressing plasmids in the cells (Fig. 1B). To further determine whether ANT2 affects sorafenib efficacy in HCC cells, we transfected Huh7 cells with the ANT2-expressing plasmids. Then, we performed an MTT assay in the cells transfected with the ANT2-expressing plasmids. The results revealed that ANT2 transformed Huh7 to Huh7-R cells (Fig. 1C), suggesting that its overexpression promotes sorafenib resistance.

To identify whether ANT2 affects RASSF1, SIRT3, NURR1, DLX2 and ADRB2, we performed western blotting to detect their expression in Huh7 cells. Our results showed that RASSF1 was downregulated and NURR1, DLX2 and ADRB2 were upregulated in the Huh7 cells transfected with ANT2 (Fig. 1D).

ANT2 promotes formation of CIC phenotypes in $\mathrm{Huh} 7 \mathrm{cells}$. In order to identify whether ANT2 affects CIC traits in Huh7 cells, we performed a sphere-forming assay to assess the ability of CIC or CIC-like cell-self renewal in Huh7 cells. The sphere-forming assay revealed that ANT2-overexpressing cells formed much bigger spheres after 14 days of culture when compared to the control cells, indicating markedly increased CIC traits by ANT2 (Fig. 2A). CD133, CD44 and EpCAM are positively associated with CIC-like characteristics in HCC (29-31). To determine whether ANT2 regulates CD133, CD44 and EpCAM protein expression, we performed western blotting in Huh7 cells transfected with the ANT2-expressing plasmids and empty vectors. The results showed that the protein expression of CD133, CD44 and EpCAM was upregulated in the Huh7 cells transfected with the ANT2-expressing plasmids (Fig. 2B). 

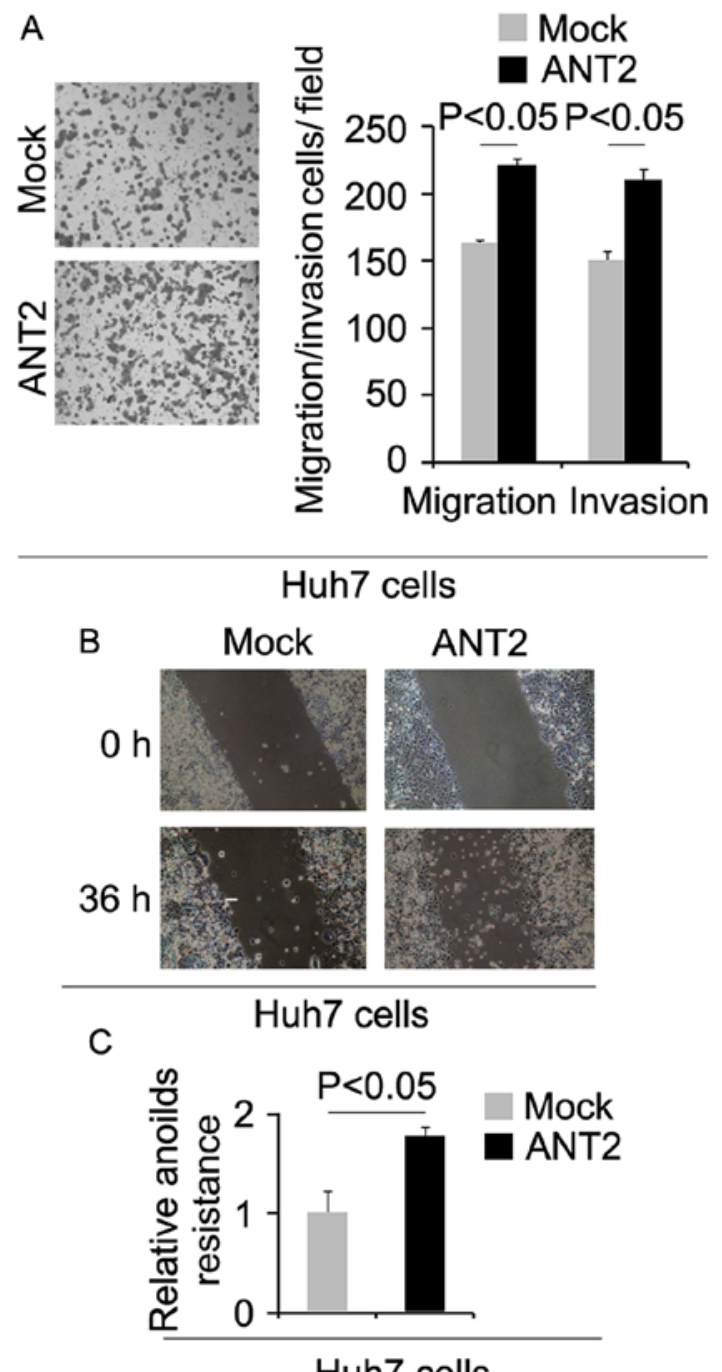

Huh7 cells

Figure 3. Adenine nucleotide translocator (ANT2) promotes metastasis-associated traits in Huh7 cells. (A) Invasion and migration assays of Huh7 cells transfected with ANT2-expressing plasmids and empty vector (mock). $n=3$. (B) Wound-healing assays of Huh7 cells transfected with ANT2-expressing plasmids and empty vector (mock). $n=3$. (C) Anoikis assays of Huh7 cells transfected as indicated. $n=3$.

ANT2 promotes metastasis-associated traits in Huh7 cells. To determine whether cells with increased CIC characteristics have increased metastatic ability, we performed migration, invasion, wound healing and anoikis assays. We found that migration, invasion and anoikis resistance were increased by ANT2 (Fig. 3A-C).

Silencing of miR-137 upregulates ANT2 protein expression in Huh7 cells. Having demonstrated that ANT2 was upregulated in Huh7-R cells and that its overexpression promoted sorafenib resistance in Huh7 cells and promoted formation of CIC phenotypes, we next studied the mechanisms regulating ANT2 expression in Huh7 cells. miRs are a class of small non-coding RNAs ( $\sim 22$ nucleotides), that negatively regulate protein-coding gene expression by targeting mRNA degradation or translation inhibition $(32,33)$.

To further confirm whether ANT2 could be regulated by microRNAs, we employed the commonly used prediction algorithm, miRanda (http://www.microrna.
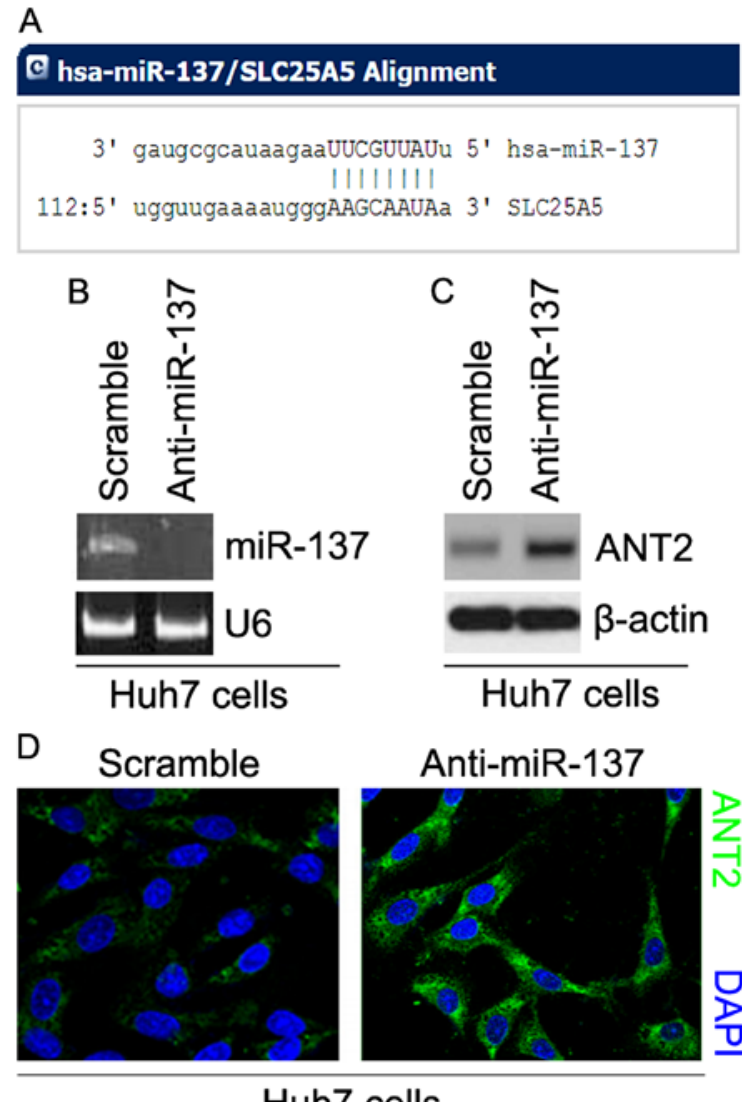

Huh7 cells

Figure 4. Silencing of miR-137 upregulates adenine nucleotide translocator (ANT2) protein expression in Huh7 cells. (A) Schematic of predicted miR-137-binding sites in the 3'UTR of ANT2 mRNA by miRanda. (B) Realtime PCR of miR-137 in Huh7 cells transfected with anti-miR-137 and scramble. U6 was used as a loading control. $n=3$. (C) Western blotting for ANT2 in Huh7 cells transfected with anti-miR-137 and scramble. $\beta$-actin was used as a loading control. $n=3$. (D) Immunofluorescence analyses for ANT2 in Huh7 cells transfected with anti-miR-137 and scramble. $n=3$.

org/microrna/home.do) to analyze the $3^{\prime}$ untranslated region ( 3 'UTR) of ANT2. Twelve miRs were identified by the algorithm. However, our interests concerned miR-137, since it has been reported that miR-137 is significantly downregulated in HCC. Its decreased expression is associated with vascular invasion, incomplete involucrum and distant metastasis (28). Target sites on the 3'UTR of ANT2 are shown in Fig. 4A. We reasoned that miR-137 downregulated ANT2 expression by targeting its 3'UTR in HCC cells. Sliencing of miR-137 contributes to the upregulation of ANT2 and sorafenib resistance in Huh7 cells.

In an attempt to identify the role of miR-137 in the regulation of ANT2 expression in Huh7 cells, we transfected Huh7 cells with anti-miR-137 and scramble. After transfection, miR-137 expression was detected by real-time PCR and the results revealed that miR-137 was significantly decreased by anti-miR-137 in the cells (Fig. 4B). To ascertain the reason, we performed western blotting to detect ANT2 protein expression in Huh7 cells transfected with anti-miR-137 and scramble. The results revealed that the protein expression of ANT2 was significantly upregulated by anti-miR-137 (Fig. 4C). We next performed immunofluorescence analyses in Huh7 cells transfected with anti-miR-137 and scramble. The results showed that the protein expression 


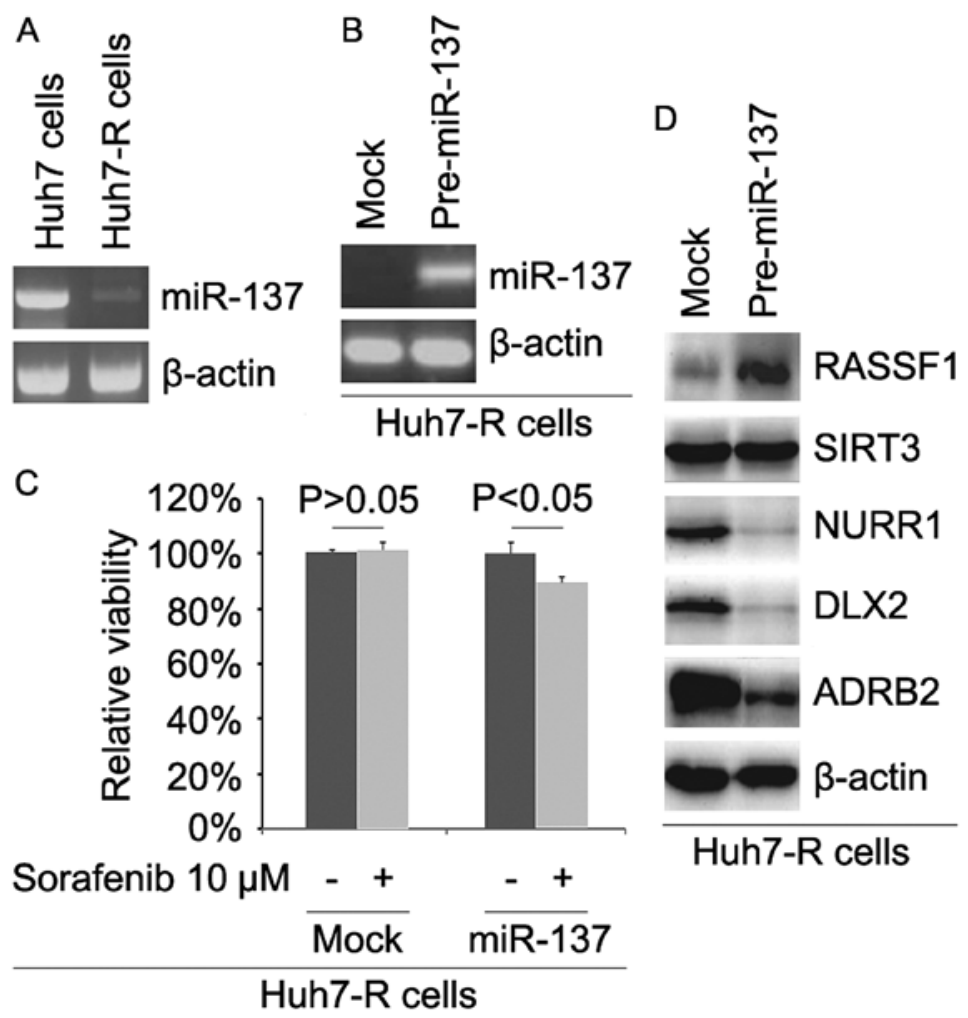

Figure 5. miR-137 is downregulated and its restoration reverses sorafenib resistance in Huh7-R cells. (A) Real-time PCR for miR-137 in Huh7 and Huh7-R cells. $\beta$-actin was used as a loading control. $n=3$. (B) Real-time PCR for miR-137 in Huh7-R cells transfected with pre-miR-137 and control miR (mock). $\beta$-actin was used as a loading control. $n=3$. (C) An MTT assay was performed for cell viability in Huh7-R cells. Huh7-R cells transfected with pre-miR-137 and control miR (mock) were untreated or treated with sorafenib. (D) Western blotting for RASSF1, SIRT3, NURR1, DLX2 and ADRB2 in Huh7 cells transfected with pre-miR-137 and control miR (mock). $\beta$-actin was a loading control. $n=3$.

of ANT2 was clearly increased in the cells transfected with anti-miR-137 (Fig. 4D).

miR-137 is downregulated and its restoration reverses sorafenib resistance in Huh7-R cells. In order to detect whether sorafenib resistance is associated with miR-137 expression, we analyzed miR-137 expression in Huh7 and Huh7-R cells. The results revealed that miR-137 expression was downregulated in the Huh7-R cells (Fig. 5A). To identify the role of miR-137, we determined whether pre-miR-137 could cause stable expression of miR-137 in Huh7-R cells. The results showed that the expression of miR-137 was significantly increased by pre-miR-137 in the cells (Fig. 5B). To further ascertain whether miR-137 affects sorafenib efficacy in HCC cells, we transfected Huh7-R cells with pre-miR-137. Then, we performed an MTT assay in Huh7-R cells transfected with pre-miR-137. The results revealed that miR-137 transformed Huh7-R to Huh7 cells (Fig. 5C), suggesting that its overexpression reversed sorafenib resistance. To identify whether miR-137 affects RASSF1, SIRT3, NURR1, DLX2 and ADRB2, we performed western blotting to detect their expression in Huh7-R cells. Our results showed that RASSF1 was upregulated and NURR1, DLX2 and ADRB2 were downregulated in the Huh7-R cells transfected with pre-miR-137 (Fig. 5D).

miR-137 inhibits formation of CIC phenotypes in Huh7-R cells. In order to identify whether miR-137 affects CIC traits in Huh7-R cells, we performed a sphere-forming assay to assess the ability of CIC or CIC-like cell self-renewal in Huh7-R

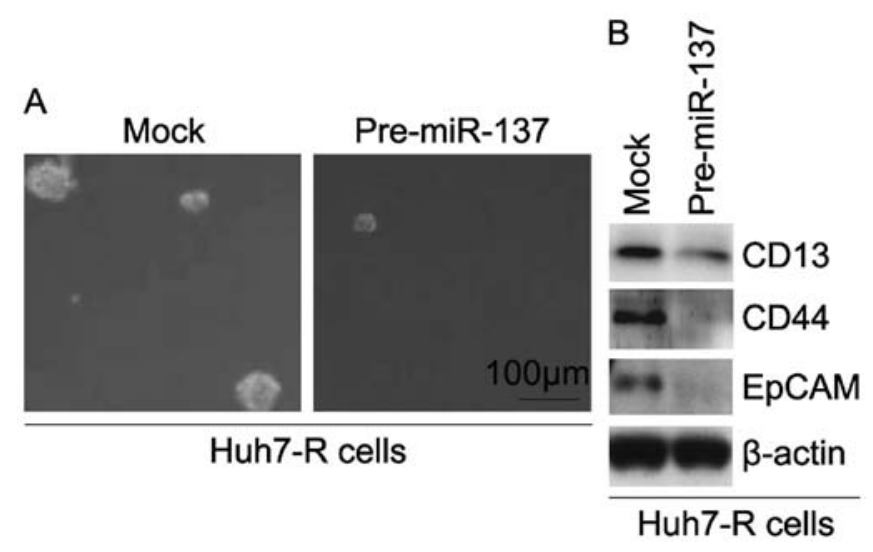

Figure 6. miR-137 inhibits the formation of cancer-initiating cell (CIC) phenotypes in Huh7-R cells. (A) Sphere growth for Huh7-R cells transfected with pre-miR-137 and control miR (mock). The data are reported as the number of spheres formed/1,000 seeded cells. $n=3$. (B) Western blotting for the protein expression of CD133, CD44 and EpCAM in Huh7-R cells transfected with pre-miR-137 and control miR (mock). $\beta$-actin was used as a loading control. $\mathrm{n}=3$.

cells. The sphere-forming assay revealed that miR-137-overexpressing cells formed much smaller spheres after 14 days of culture as compared with the control cells, indicating markedly increased CIC traits by miR-137 (Fig. 6A). To determine whether miR-137 regulates the protein expression of CD133, CD44 and EpCAM, we performed western blotting in Huh7-R cells transfected with pre-miR-137 and control miR. The results revealed that the protein expression of CD133, CD44 
A
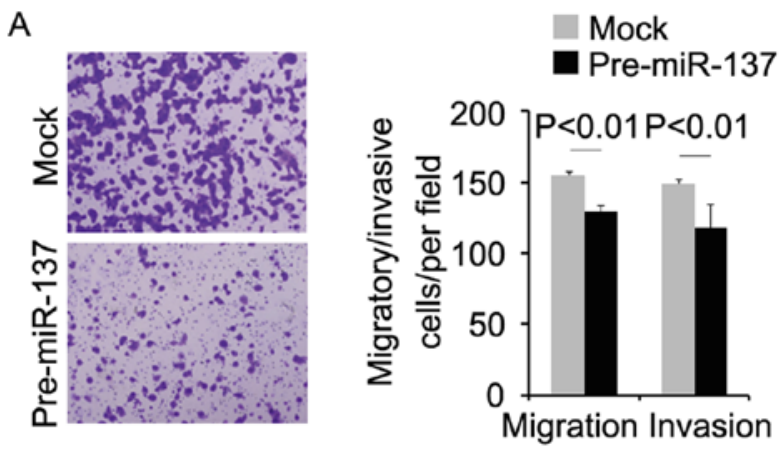

B

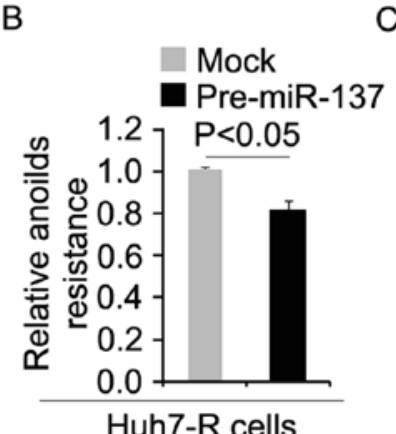

Huh7-R cells

C

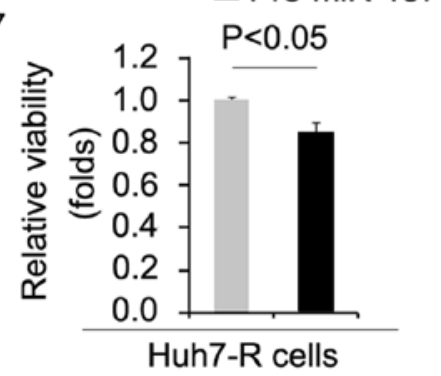

Figure 7. miR-137 inhibits metastasis-associated traits in Huh7-R cells. (A) Invasion and migration assays for Huh7-R cells transfected with pre-miR-137 and control miR (mock). $n=3$. (B) Anoikis assays for Huh7-R cells transfected with pre-miR-137 and control miR (mock). n=3. (C) An MTT assay was performed for cell viability in Huh7-R cells. Huh7-R cells were transfected with pre-miR-137 and control miR (mock).

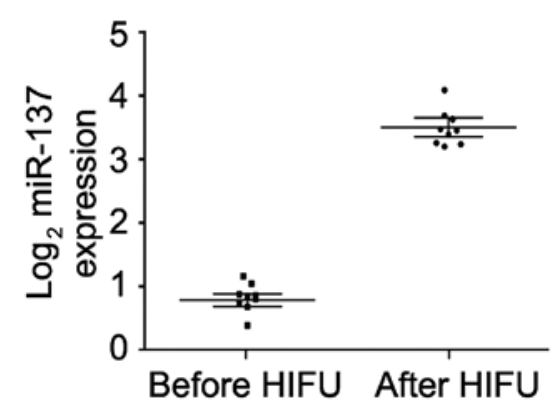

Figure 8. High-intensity focused ultrasound (HIFU) promotes serum miR-137 expression in unresectable HCC patients.

and EpCAM was downregulated in the Huh7-R cells transfected with pre-miR-137 (Fig. 6B).

miR-137 inhibits metastasis-associated traits in Huh7-R cells. To determine whether cells with decreased CIC characteristics had attenuated metastatic ability, we performed migration, invasion and anoikis assays. We found that migration, invasion and anoikis resistance were inhibited by pre-miR-137 in the Huh7-R cells (Fig. 7A and B). Moreover, in order to detect whether miR-137 affects proliferation, we performed an MTT assay in the Huh7-R cells. We found that its overexpression inhibited proliferation in the Huh7-R cells (Fig. 7C).

HIFU promotes serum miR-137 expression in unresectable HCC patients. In order to determine whether HIFU affects

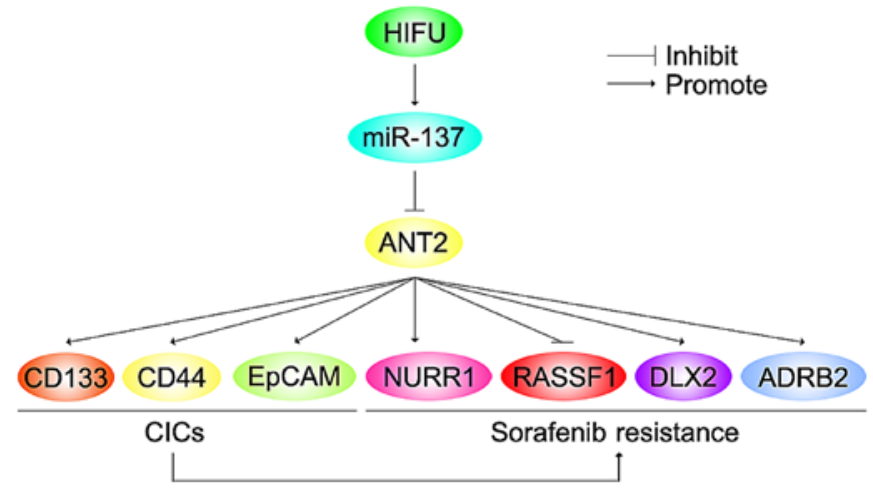

Figure 9. Upregulation of miR-137 reverses sorafenib resistance and cancer-initiating cell (CIC) phenotypes by degrading adenine nucleotide translocator (ANT2) in hepatocellular carcinoma.

serum miR-137 expression in unresectable HCC patients, we recruited 9 unresectable HCC patients who received HIFU treatment. Real-time PCR was performed to compare the difference in serum miR-137 before and after treatment. We found that receiving HIFU significantly promoted miR-137 expression in the serum of these patients (Fig. 8).

\section{Discussion}

Sorafenib, a multikinase inhibitor, is the only standard clinical drug used against patients with advanced hepatocellular carcinoma (HCC). However, development of sorafenib resistance in HCC often prevents its long-term efficacy. Therefore, novel targets and strategies are urgently needed to improve the antitumor effect of sorafenib.

miR-137 expression is significantly downregulated in HCC. Its decreased expression is associated with vascular invasion, incomplete involucrum and distant metastasis (34). Decreased miR-137 expression is an independent indicator for poor survival (34). Overexpression of miR-137 suppresses cell proliferation, migration and invasion in vitro (34). Consistent with the previous studies, we demonstrated that decreased miR-137 expression is associated with sorafenib resistance in HCC Huh7 cells. Current treatment cannot eliminate cancer-initiating cells (CICs) $(2,3)$. This is a cause for anticancer drug resistance and recurrence. We found that miR-137 inhibited formation of CIC traits as well as migration and invasion in Huh7-R cells. RASSF1 is a MAPK signaling factor and knockdown of RASSF1 increased sorafenib resistance (35). Knockdown of NURR1 significantly increased cell sensitivity to sorafenib and inhibited the cell growth, migration and invasion of HCC cells, both in vitro and in vivo (36). DLX2 facilitates sorafenib resistance by promoting the expression of markers of epithelial-mesenchymal transition and by activating the extracellular signal-regulated protein kinase pathway (37). ADRB2 signaling promotes HCC progression and sorafenib resistance by inhibiting autophagic degradation of HIF1 $\alpha$ (38). We demonstrated that RASSF1 expression can be induced and NURR1, DLX2 and ADRB2 can be inhibited by miR-137. The results indicate that miR-137 may be a therapeutic target and a reliable biomarker that can be used to predict sorafenib resistance and ensure more effective clinical management. 
ANT2 suppression by shRNA exerts anticancer effects in HCC by regulating different pathways (17-19). However, its regulatory mechanism has yet to be reported. We found that silencing of miR-137 significantly upregulated ANT2 protein expression in Huh7 cells. Moreover, we demonstrated that ANT2 was upregulated in sorafenib-resistant HCC Huh7 cells (Huh7-R) and its overexpression promoted sorafenib resistance. ANT2 induced the formation of CIC phenotypes and promoted metastasis-associated traits in the Huh7 cells. We demonstrated that ANT2 overexpression inhibited RASSF1 expression and promoted NURR1, DLX2 and ADRB2 expression in Huh7 cells. In the present study, we discovered the role for ANT2 in HCC, its association with sorafenib resistance, and the antitumor effects of a newly identified microRNA, miR-137, that targets ANT2. These findings have potential therapeutic significance as testing for the absence of ANT2 at diagnosis may be helpful for identifying patients who are likely to have a response to sorafenib.

HIFU is the latest developed local ablation technique for unresectable HCC (20). We found that after receiving HIFU, miR-137 expression was upregulated in the serum of patients with unresectable HCC (Fig. 9). Thus, combining HIFU and sorafenib may be a wise option for advanced and unresectable HCC (Fig. 9).

\section{References}

1. Cervello M, McCubrey JA, Cusimano A, Lampiasi N, Azzolina A and Montalto G: Targeted therapy for hepatocellular carcinoma: Novel agents on the horizon. Oncotarget 3: 236-260, 2012.

2. Dean M, Fojo T and Bates S: Tumour stem cells and drug resistance. Nat Rev Cancer 5: 275-284, 2005.

3. Zhang Q, Shi S, Yen Y, Brown J, Ta JQ and Le AD: A subpopulation of $\mathrm{CD} 33^{+}$cancer stem-like cells characterized in human oral squamous cell carcinoma confer resistance to chemotherapy. Cancer Lett 289: 151-160, 2010.

4. Llovet JM, Ricci S, Mazzaferro V, Hilgard P, Gane E, Blanc JF, de Oliveira AC, Santoro A, Raoul JL, Forner A, et al; SHARP Investigators Study Group: Sorafenib in advanced hepatocellular carcinoma. N Engl J Med 359: 378-390, 2008.

5. Di Maio M, Daniele B and Perrone F: Targeted therapies: Role of sorafenib in HCC patients with compromised liver function. Nat Rev Clin Oncol 6: 505-506, 2009.

6. Bioulac-Sage P, Laumonier H, Couchy G, Le Bail B, Sa Cunha A, Rullier A, Laurent C, Blanc JF, Cubel G, Trillaud H, et al: Hepatocellular adenoma management and phenotypic classification: The Bordeaux experience. Hepatology 50: 481-489, 2009.

7. Liu P, Cheng H, Roberts TM and Zhao JJ: Targeting the phosphoinositide 3-kinase pathway in cancer. Nat Rev Drug Discov 8 . 627-644, 2009

8. Scanga A and Kowdley K: Sorafenib: A glimmer of hope for unresectable hepatocellular carcinoma? Hepatology 49: 332-334, 2009.

9. Wilhelm SM, Carter C, Tang L, Wilkie D, McNabola A, Rong H, Chen C, Zhang X, Vincent P, McHugh M, et al: BAY 43-9006 exhibits broad spectrum oral antitumor activity and targets the RAF/MEK/ERK pathway and receptor tyrosine kinases involved in tumor progression and angiogenesis. Cancer Res 64: 7099-7109, 2004.

10. Panka DJ, Wang W, Atkins MB and Mier JW: The Raf inhibitor BAY 43-9006 (Sorafenib) induces caspase-independent apoptosis in melanoma cells. Cancer Res 66: 1611-1619, 2006.

11. Adnane L, Trail PA, Taylor I and Wilhelm SM: Sorafenib (BAY 43-9006, Nexavar), a dual-action inhibitor that targets $\mathrm{RAF} / \mathrm{MEK} / \mathrm{ERK}$ pathway in tumor cells and tyrosine kinases VEGFR/PDGFR in tumor vasculature. Methods Enzymol 407: 597-612, 2006

12. Wilhelm S, Carter C, Lynch M, Lowinger T, Dumas J, Smith RA, Schwartz B, Simantov R and Kelley S: Discovery and development of sorafenib: A multikinase inhibitor for treating cancer. Nat Rev Drug Discov 5: 835-844, 2006.
13. Zhang W, Konopleva M, Shi YX, McQueen T, Harris D, Ling X, Estrov Z, Quintás-Cardama A, Small D, Cortes J, et al: Mutant FLT3: A direct target of sorafenib in acute myelogenous leukemia. J Natl Cancer Inst 100: 184-198, 2008.

14. Cheng AL, Kang YK, Chen Z, Tsao CJ, Qin S, Kim JS, Luo R, Feng J, Ye S, Yang TS, et al: Efficacy and safety of sorafenib in patients in the Asia-Pacific region with advanced hepatocellular carcinoma: A phase III randomised, double-blind, placebocontrolled trial. Lancet Oncol 10: 25-34, 2009.

15. Schönfeld P, Schild L and Bohnensack R: Expression of the ADP/ATP carrier and expansion of the mitochondrial (ATP + ADP) pool contribute to postnatal maturation of the rat heart. Eur J Biochem 241: 895-900, 1996.

16. Chevrollier A, Loiseau D, Reynier P and Stepien G: Adenine nucleotide translocase 2 is a key mitochondrial protein in cancer metabolism. Biochim Biophys Acta 1807: 562-567, 2011.

17. Jang JY, Jeon YK, Lee CE and Kim CW: ANT2 suppression by shRNA may be able to exert anticancer effects in HCC further by restoring SOCS1 expression. Int J Oncol 42: 574-582, 2013.

18. Baik SH, Lee J, Lee YS, Jang JY and Kim CW: ANT2 shRNA downregulates miR-19a and miR-96 through the PI3K/Akt pathway and suppresses tumor growth in hepatocellular carcinoma cells. Exp Mol Med 48: e222, 2016.

19. Jang JY, Lee YS, Jeon YK, Lee K, Jang JJ and Kim CW: ANT2 suppression by shRNA restores miR-636 expression, thereby downregulating Ras and inhibiting tumorigenesis of hepatocellular carcinoma. Exp Mol Med 45: e3, 2013.

20. Ng KK, Poon RT, Chan SC, Chok KS, Cheung TT, Tung H, Chu F, Tso WK, Yu WC, Lo CM, et al: High-intensity focused ultrasound for hepatocellular carcinoma: A single-center experience. Ann Surg 253: 981-987, 2011.

21. Liao XH, Li YQ, Wang N, Zheng L, Xing WJ, Zhao DW, Yan TB, Wang Y, Liu LY, Sun XG, et al: Re-expression and epigenetic modification of maspin induced apoptosis in MCF-7 cells mediated by myocardin. Cell Signal 26: 1335-1346, 2014.

22. Liao XH, Wang Y, Wang N, Yan TB, Xing WJ, Zheng L, Zhao DW, Li YQ, Liu LY, Sun XG, et al: Human chorionic gonadotropin decreases human breast cancer cell proliferation and promotes differentiation. IUBMB Life 66: 352-360, 2014.

23. Liao XH, Xiang Y, Yu CX, Li JP, Li H, Nie Q, Hu P, Zhou J and Zhang TC: STAT3 is required for MiR-17-5p-mediated sensitization to chemotherapy-induced apoptosis in breast cancer cells. Oncotarget: Feb 2, 2017 (Epub ahead of print).

24. Liao XH, Li JY, Dong XM, Wang X, Xiang Y, Li H, Yu CX, Li JP, Yuan BY, Zhou J, et al: ER $\alpha$ inhibited myocardin-induced differentiation in uterine fibroids. Exp Cell Res 350: 73-82, 2017.

25. Liao XH,Lu DL, Wang N,Liu LY, Wang Y, Li YQ, Yan TB, Sun XG, $\mathrm{Hu} \mathrm{P}$ and Zhang TC: Estrogen receptor $\alpha$ mediates proliferation of breast cancer MCF-7 cells via a p21/PCNA/E2F1-dependent pathway. FEBS J 281: 927-942, 2014.

26. Xiang Y, Lu DL, Li JP, Yu CX, Zheng DL, Huang X, Wang ZY, $\mathrm{Hu}$ P, Liao XH and Zhang TC: Myocardin inhibits estrogen receptor alpha-mediated proliferation of human breast cancer MCF-7 cells via regulating MicroRNA expression. IUBMB Life 68: 477-487, 2016.

27. Ren ZG, Dong SX, Han P and Qi J: miR-203 promotes proliferation, migration and invasion by degrading SIK1 in pancreatic cancer. Oncol Rep 35: 1365-1374, 2016.

28. Lu Y, Chopp M, Zheng X, Katakowski M, Buller B and Jiang F: MiR-145 reduces ADAM17 expression and inhibits in vitro migration and invasion of glioma cells. Oncol Rep 29: 67-72, 2013.

29. Suetsugu A, Nagaki M, Aoki H, Motohashi T, Kunisada T and Moriwaki H: Characterization of $\mathrm{CD}_{133^{+}}$hepatocellular carcinoma cells as cancer stem/progenitor cells. Biochem Biophys Res Commun 351: 820-824, 2006.

30. Zhu Z, Hao X, Yan M, Yao M, Ge C, Gu J and Li J: Cancer stem/progenitor cells are highly enriched in CD $133^{+} \mathrm{CD} 44^{+}$ population in hepatocellular carcinoma. Int J Cancer 126: 2067-2078, 2010.

31. Yamashita T, Ji J, Budhu A, Forgues M, Yang W, Wang HY, Jia H, Ye Q, Qin LX, Wauthier E, et al: EpCAM-positive hepatocellular carcinoma cells are tumor-initiating cells with stem/progenitor cell features. Gastroenterology 136: 1012-1024, 2009.

32. Lee RC, Feinbaum RL and Ambros V: The C. elegans heterochronic gene lin-4 encodes small RNAs with antisense complementarity to lin-14. Cell 75: 843-854, 1993.

33. Pasquinelli AE, Reinhart BJ, Slack F, Martindale MQ, Kuroda MI, Maller B, Hayward DC, Ball EE, Degnan B, Müller P, et al: Conservation of the sequence and temporal expression of let-7 heterochronic regulatory RNA. Nature 408: 86-89, 2000. 
34. Liu LL, Lu SX, Li M, Li LZ, Fu J, Hu W, Yang YZ, Luo RZ, Zhang CZ and Yun JP: FoxD3-regulated microRNA-137 suppresses tumour growth and metastasis in human hepatocellular carcinoma by targeting AKT2. Oncotarget 5: 5113-5124, 2014.

35. Azumi J, Tsubota T, Sakabe T and Shiota G: miR-181a induces sorafenib resistance of hepatocellular carcinoma cells through downregulation of RASSF1 expression. Cancer Sci 107: 1256-1262, 2016.

36. Emma MR, Iovanna JL, Bachvarov D, Puleio R, Loria GR, Augello G, Candido S, Libra M, Gulino A, Cancila V, et al: NUPR1, a new target in liver cancer: Implication in controlling cell growth, migration, invasion and sorafenib resistance. Cell Death Dis 7: e2269, 2016.
37. Liu J, Cui X, Qu L, Hua L, Wu M, Shen Z, Lu C and Ni R: Overexpression of DLX2 is associated with poor prognosis and sorafenib resistance in hepatocellular carcinoma. Exp Mol Pathol 101: 58-65, 2016.

38. Wu FQ, Fang T, Yu LX, Lv GS, Lv HW, Liang D, Li T, Wang CZ, Tan YX, Ding J, et al: ADRB2 signaling promotes HCC progression and sorafenib resistance by inhibiting autophagic degradation of HIF1 $\alpha$. J Hepatol 65: 314-324, 2016. 